Int. J. Dev. Biol. 48: 701-705 (2004)

doi: $10.1387 / \mathrm{ijdb} .041888 \mathrm{rf}$

\title{
Evolving eyes
}

\author{
RUSSELL D. FERNALD* \\ Biological Sciences and Neuroscience Program, Stanford University, Stanford, CA, USA
}

\begin{abstract}
Despite the incredible diversity among extant eyes, laws of physics constrain how light can be collected resulting in only eight known optical systems in animal eyes. Surprisingly, all animal eyes share a common molecular strategy using opsin for catching photons, but there are a diverse collection of mechanisms with proteins unrelated to each other used to focus light for vision. However, opsin is expressed in either one of two types of photoreceptor that differ fundamentally in their structure and tissue of origin. Taken together, this collection of observations strongly suggests that eyes have had multiple origins with remarkable convergence due to physics and molecular conservation of the opsin protein. Yet recent work has shown that a family of conserved genes are involved in eye formation despite substantial differences in their structure and origin, leading to a controversy over whether eyes evolved once or repeatedly. A likely resolution of this discussion is that particular genes and genetic programs have become associated with specific features needed for eyes and such suites of genes have been recruited as new eyes evolve. Since specific genes and their products are used repeatedly, it is somewhat difficult to conceptualize their causal relationships relative to evolutionary processes. However, detailed comparison of developmental programs may offer clues about multiple origins.
\end{abstract}

KEY WORDS: vertebrate eye, development, eye evolution, eye variety

\section{Introduction}

Light has been the dominant selective force on our planet, resulting in circadian rhythms and the ubiquitous biological clock as well as the most important organ, the eye. Scientists have always been fascinated by eyes, wondering about their remarkable variety, their exquisite functional properties, their development and ultimately their evolutionary origin. As a result, we know a lot about variety in eyes, morphological sources of ocular tissue, some molecular actors responsible for eye development and even about how eyes might have evolved. This review will present highlights of these topics along with some ideas about how to think about the evolution of eyes.

\section{Eye Variety}

In his landmark book, Walls (1942) showed that the variety of eyes is quite astonishing and included a staggering range of adaptations produced by selective pressures for vision in different visual habitats. However, there are several features common to all eyes as a result of constraints on their construction. Since eyes collect and focus light, limits on their structure depend on the physical properties of light, which, in turn, sets limits on the optical features of eyes (c.f., Fernald, 1988, Fig. 1). For example, eyes have evolved to be sensitive to a narrow range of wavelengths relative to the broad spectrum of energy produced by sunlight. This is likely because early evolution occurred in water which strongly filters light (Fernald 1988). So the narrow range of sensitivity probably resulted from selection for biochemical mechanisms sensitive to the range of wavelengths that could penetrate water and set the stage for wavelength sensitivity during evolution. Many animal species have long since moved onto land and are exposed to the broader spectrum of EM radiation from the sun, but most animal eyes remain limited to seeing within this narrow band. Insects and some species of fish and birds later evolved receptors in ultraviolet region (e.g. Viltala et al., 1995) but the limited range of wavelength sensitivity reflects our aquatic origins, an evolutionary adaptation that persists.

Of ca. 33 phyla, about a third have no specialized organ for detecting light, a third have light sensitive organs and the remainder have animals with what we would consider eyes (Land \& Nilsson, 2002). Image-forming eyes evolved in six of the 33 extant metazoan phyla (Cnidaria, Mollusca, Annelida, Onychophora, Arthropoda, and Chordata), and these six contribute about $96 \%$ of the known species alive today (Land and Fernald, 1992), suggesting eyes are indeed useful. Existing eyes reflect diverse solutions to the problem of obtaining an image and range in size from a fraction of a millimeter to tens of centimeters in diameter. The range of eye

\footnotetext{
*Address correspondence to: Dr. Russell D. Fernald. Department of Biological Sciences, Gilbert Hall, Stanford University, Stanford CA 94305, USA. Fax: +1-650-723-0881. e-mail: rfernald@stanford.edu
} 
types, sizes and locations suggests that they can evolve relatively easily (see below).

Optical systems fall into three classes based on their image forming mechanisms: Images formed via shadows, images formed via refraction (e.g. lens \&/or cornea), and images formed via reflection. These different optical types were first systematically described by Land (1981) who has contributed significantly to our understanding of eyes and particularly to their optical function (e.g., Fig. 1.9, Land \& Nilsson, 2002). As noted above, physical laws fundamentally limit how an eye can be built, whether it produces an image or simply records the direction of incident light. For this reason, similar structures have arisen convergently in distinctly unrelated animals such as fish and cephalopods. The chambered or camera eyes in these two lineages are similar in a very large number of details, despite the fact that their owners are phylogenetically distant (Packard, 1972). Both evolved spherical lenses to achieve sufficient refractive power for vision underwater but the inverted retinal layers of the fish (and all vertebrate retinas) are distinctly different from the non-inverted, somewhat simpler retina of squid. Macroscopically, these eye types and the animals bearing them are non homologous, even though there are homologies at the molecular and developmental levels which is at the heart of understanding eye evolution.

Invertebrates have the greatest variety of eye types with both camera eyes (e.g. Cephalopods) and compound eyes. Moreover, the number and location on given species is also most varied in invertebrates. While vertebrates settled on paired, chambered eyes with lenses, invertebrate species may have multiple non-paired eyes and eyes in remarkable locations. For example, certain butterflies have light detecting organs located such that darkness signals successful copulation (Arikawa et al., 1996). And recently Nilsson and colleagues (Nordström et al., 2003), described a visual system in the planula of a box jellyfish Tripedalia cystophora with eyecups directly connected to motor cilium. That is, no nervous system to process information because the eyes are a complete sensory-motor system unto themselves.

While primitive eyes may provide information about intensity and possibly direction of a light source, advanced eyes collect information about wavelength, contrast and can, as in many vertebrates, provide high resolution images of the illuminated scene from a concentration of receptors in the fovea. The capabilities of eyes as a function of their structure and hence the different specificity of their developmental programs varies greatly. For example, resolution of an image as measured in subtended degrees differs by ca. 13-fold among vertebrates with the eagle being most acute with an acuity ca. 10,000 fold greater than planaria (Land \& Nilsson, 2002). Similarly, comparing sensitivities reveals a range of $4 \times 10^{5}$ between deep sea animals and human foveal vision (Land \& Nilsson, 2002).

The selective pressures for evolution of wavelength discrimination appear to have been quite pervasive. Very likely the selective pressure favored better contrast discrimination increasing the likelihood of finding food, mates and predators would have been enhanced with chromatic information (e.g. Nagle and Osorio, 1993; Osorio and Vorobyev, 1996). Indeed, recent work comparing eight primate taxa suggest that trichromatic vision evolved where leaf consumption was critical (Lucas et al., 2003). In support of this idea, many species of diurnal reptiles and birds have colored retinal filters composed of oil droplets that appear to have evolved to increase the number of colors that can be discriminated, suggesting selective pressure for improved color vision (Vorobyev, 2003).
Molecules that detect light, opsins appeared in biological systems before eyes (Land and Fernald, 1992) and evolved along at least five lines diverging from an ancestral type before teleost fish diverged from other vertebrates (e.g. Hisatomi et al., 1994). That visual pigments evolved along parallel lines following an ancient divergence is widely accepted though there are some differences in interpretation (Okano et al., 1992). Primate photopigments offer examples of recent evolutionary change in these important molecules. Old world monkeys, apes and humans have trichromatic vision, while New World monkeys are polymorphic, having dichromatic or trichromatic color vision (Jacobs, 1996). Humans seem unique in their polymorphism of trichromacy (e.g. Neitz et al., 1996), which might reflect the absence of a potent selective pressure. The subtlety of the selective pressure on chromatic detection is evident from variation within a species of bluefin killifish where the relative abundance of cone types depends on whether the animals live in springs or swamps (Fuller etal., 2003). The novel differential spectral sensitivity between these populations is produced through differential expression of cone classes in the retina rather than via modification of the spectral tuning of opsin molecules showing that there are different ways to achieve color detection.

This small selection of examples shows that animals have evolved eyes with resolution, sensitivity and wavelength sensitivities to match their needs. Most of those adaptations are the result of developmental differences as eyes are built during embryogenesis. What is known about the genes responsible for producing the differences that are so functionally important?

\section{Lenses: another view of the evolution of eyes}

The vertebrate eye develops from a diverse collection of embryonic sources through a complex set of inductive events (reviewed in Graw, 1996). Whereas the neural retina is derived from the diencephalon and is a part of the brain, the lens comes from surface ectoderm and the iris and ciliary body arise primarily from the neural crest. Mapping the genes known to play a role in mouse eye development, for example, shows that some of these genes are present on every chromosome (see Graw, 1996; Fig. 2). The apparent patchwork assembly of the eye makes it all the more surprising that common developmental programs seem to produce comparable outcomes across a broad phylogenetic divide (Halder et al., 1995). Could we use the phylogeny of conserved lens proteins to gain insight into eye evolution?

Vertebrate lenses are formed from modified epithelial cells, that contain high concentrations of soluble proteins known as crystallins because they are packed in a highly organized fashion. It is the change in relative concentration of these proteins from the periphery to the center of vertebrate lenses that produces the refractive index gradient necessary for a lens to be useful to the animal. In fact, the identity of the proteins seems not to be important since the crystallin proteins are not more transparent than others. Instead the distribution of protein concentration as a function of radius is the key to a successful lens. Thus the challenge in understanding lens evolution lies in discovering how the distribution of proteins within a lens is established and maintained.

Of the eleven lens crystallins now known, only three, $\alpha, \beta$ and $\gamma$ crystallins are ubiquitous among vertebrates. In fact, until recently, all crystallins were thought to be unique to lens tissue and to have evolved for this special function. However, despite their apparently 
specialist role, most of the crystallins are neither structural proteins nor lens specific. There are two major groups of lens crystallins, those present in all vertebrates and those specific to a particular taxon. For example, in crocodiles and some bird species, the glycolytic enzyme lactate dehydrogenase B is a major protein in the lens. Indeed, four of the eight taxon-specific crystallins are identical to metabolic enzymes and products of the same genes, suggesting these products share a gene. Recent discussions of gene sharing and the ways that gene regulation may play a role shed some light on possible evolutionary processes (Piatigorsky, 2003).

Why might enzymes be recruited to make vertebrate lenses? Perhaps the robust regulation of enzyme production is advantageous for producing sufficient protein for a lens (Wistow, 1993), but there is not much beyond speculation to support this notion. There may be some deeper reason, however, because this molecular opportunism seemed such a good idea, mollusks independently evolved the same strategy (Doolittle, 1988). Squids have lenses whose protein content is nearly entirely the enzyme glutathione S-transferase. This convergence of molecular strategy suggests that enzymes as lenses may have a functional meaning, or because it is easy to get lens cells to make a lot of enzyme, or there may be other reasons not yet understood.

\section{Molecular actors - how do they relate to macroscopic action?}

For eyes, understanding the genetic control of eye construction is particularly important because about half the cases of blindness in children have a genetic basis (Graw, 2003). The morphological changes in eye development has been seen as a set of steps toward a final tissue formation and arrangement. Well described at a macroscopic level as a sequence of large scale events, the complexity of genes involved and complexity of that expression is only slowly coming to light. Gene expression is tightly regulated and specific gene products are used repeatedly with substantial overlap in both time and space, so thinking about direct causal relationships is difficult. Nonetheless, progress in characterizing some genes responsible for particular steps in eye development has been reasonably rapid as shown in several recent reviews (Harland, 2000; Chow \& Lang, 2001; Graw, 2003). Functions for at least 15 transcription factors and several signaling molecules have been described in human and mice eyes based on developmental disorders and/or molecular manipulations (e.g., Graw, 2003). As has been the case for other molecular actors, both the transcription factors and the signaling molecules are expressed during ocular development as well as in a wide range of other tissues. This suggests that the particular combination of expression patterns are important for the properfunctioning of these genes in eye development.

As is now well known, the paired box gene 6 ( $P A X 6$ ), a member of the family of genes that encode transcription factors with a homeodomain and a paired domain appears to be important in eye formation across many species. The remarkable demonstration that $P A X 6$ could induce eyes where they shouldn't be ('ectopic') in Drosophila(Halder et al., 1995) and similar subsequent demonstration in vertebrates (Chow et al., 1999) led to the suggestion that there might be 'master control genes' responsible for development and differentiation of ocular tissue in many species. Subsequent work has suggested that master control gene is a misnomer since a suite of genes are required collectively to initiate eye development and transcription factors are a necessary part of the process. Moreover, as noted above, the genes in question actually have dynamic spatial and temporal expression during many stages of eye development in addition to expression for essential purposes in other tissues. Nonetheless, it is remarkable that some of the same genes appear in the context of eye development despite great evolutionary distance among the owners of the eyes. How did this happen?

For Drosophila eyes, it is now known that a collection of seven genes encoding transcription factors and two signaling molecules collaborate to make eyes (reviewed in Kumar, 2001). These nuclear factors (eyeless (ey), twin of eyeless (toy)-\{both of which are PAX6 homologs\}, sine oculus (so), eyes absent (eya), dachshund (dac), eye gone (eyg) and optix) and the signaling systems including the Notch and receptor tyrosine kinase pathways act via a complex regulatory network that is reasonably well

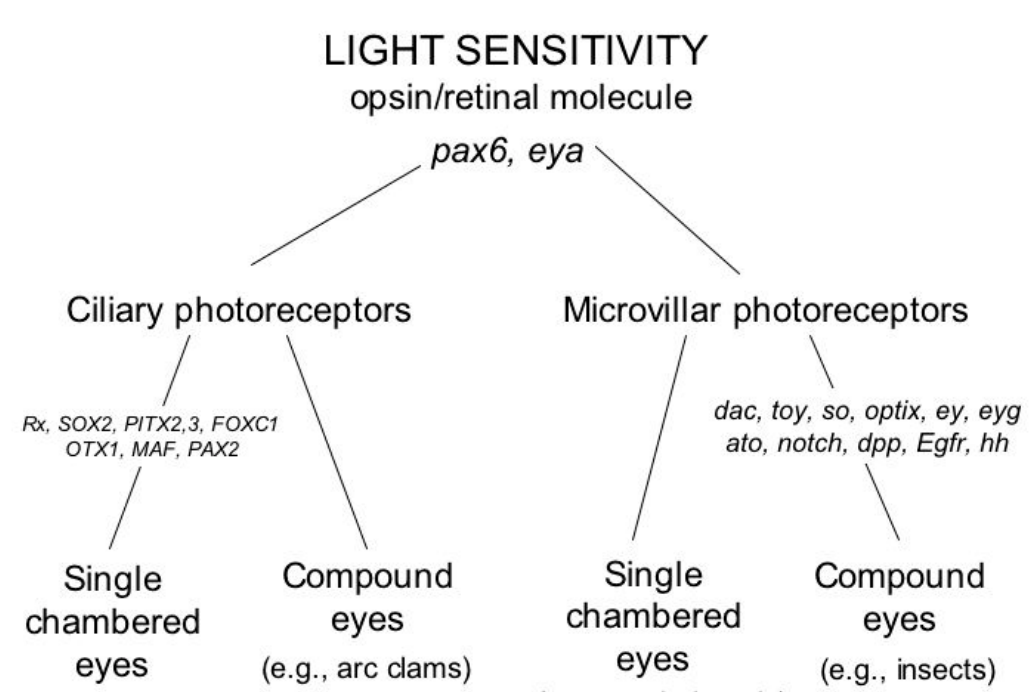

(e.g.,vertebrates)

(e.g., cephalopods)

Fig. 1. Schematic illustration of a possible scheme for the evolution of eyes indicating that all eyes share the method of capturing light (opsin) but as different eye types evolved, different suites of genes were recruited for the production of eyes (adapted from Land and Nilsson, 2002, Fig. 1.8). Following the evolution of the opsin/retinal light detection unit, its use appears to have become associated with pax 6 and possibly eya. The evolution of ciliary and microvillar photoreceptors each required recruitment of additional genes, some of which have proven to be common to one another. The identification of genes involved is not exhaustive and is based on (Kumar, 2001; Chow \& Lang, 2001; Graw, 2003). Abbreviations: ato-atonal bHLH transcription factor, dac-dachshund, dppdecapentaplegic TGF-fl secreted morphogen, Egfr-receptor tyrosine kinase, eyeyeless homeodomain/paired domain, eya-eyes absent, eyg-eye gone homeodomain, FOXC1-forkhead box gene C1, hh-hedgehog secreted morphogen, MAF-musculoaponeurotic fibrosarcoma oncogene, notch-transmembrane receptor, optix-homeodomain/six domain, OTX1-orthodenticle homologue 1, PAX2-paired box gene 2, pax6-paired domain, homeodomain, Pitx2,3-paired-like homeodomain transcription factor, Rx-retina and anterior neural fold homeobox, so-sine oculus homeodomain/six domain, SOX2-SRY-box with gene 2, toy-twin of eyeless. 
understood (Kumar, 2001; Fig. 1). The master gene hypothesis is, however, not supported because deletion of any of these genes causes loss or radical reduction in the Drosophila compound eye and, surprisingly any gene except sine oculus can cause ectopic expression of an eye in a limited set of imaginal discs in collaboration with certain signaling molecules. This means that the whole troupe of genes is needed collectively to produce a reasonable eye. Why this might be so is suggested by recent work showing that the eyagene products are phosphatases, the first case in which a transcription factor can itself dephosphorylate other proteins to fine tune gene expression ( $\mathrm{Li}$ et al., 2003). This elegant work demonstrated the details of interactions among Six 1, Dachand Eyain the formation of the kidney, muscle, inner ear as well as eyes, suggesting that this suite of genetically interacting proteins has been recruited repeatedly during evolution for organogenesis of different types.

Although it is difficult to abandon the heuristic of hierarchically organized regulatory processes in development, molecular analysis of eye development shows that this concept may not be useful. The linear sequence of genes expressed during development was originally proposed by Lewis to characterize homeotic properties of bithorax and antennapedia genes. But the molecular control of eye development needs new ways of thinking about how complex tissues are made and how such organs arose during evolution. The widespread use and redundant activities of specific genes during ocular development (e.g. Baumer et al., 2003; Chauhan et al., 2002) suggest that hierarchies, if they exist, are unknown and the more likely scenario is the orchestrated activity of a suite of molecular actors.

\section{What does development tell us about the evolution of eyes?}

Based on paleontological evidence, eyes are thought to have evolved independently in different organisms at least 40 times and possibly as many as 65 times (Salvini-Plawen and Mayr, 1977), confirming their importance to animals. Also, within arthropods, there is molecular phylogenetic evidence for independent evolutionary origin of ostracod eyes (Oakley \& Cunningham, 2002).

Explosivespeciation, or the 'Big Bang' of animal evolution happened during the Cambrian (Conway-Morris, 1998) when existing eye types improved radically, coincident with the appearance of carnivory and predation. Many selective forces were likely at work (Fernald, 2000) including use of light in behavioral signals (Parker, 1998) so no predominant selective force can be claimed. The rapidity of eye evolution has always been a question but using a simulation, Nilsson and Pelger (1994) suggested that about 2000 sequential changes could produce a typical image forming eye from a light sensitive patch. With reasonable estimates, this suggests that an eye could evolve in less than half a million years making the virtual explosion of eyes during the Cambrian seem reasonable (Land and Nilsson, 2002). After the Cambrian, arthropods, mollusks and chordates emerged with image forming eyes. Three phyla emerged from the Cambrian, with good eyes: mollusks, arthropods and chordates. Although these groups all use the opsin molecule to capture light, details of the development and structure of their eyes differ considerably.

One of the most interesting developmental differences among extant eyes is the embryonic origin of the different structures in the vertebrate and cephalopod eyes (summarized in Nilsson, 1996). The cephalopod eye forms from an epidermal placode through successive infoldings whereas the vertebrate eye emerges from the neural plate and induces the overlying epidermis to form the lens as described above. It is also noteworthy that some cephalopod eyes have a cornea while others do not while corneas are present in all vertebrates whether aquatic or not. Cephalopods which do not have a cornea have an eyelid and in those species with a cornea it is essentially an eyelid that has become transparent, turgid and permanently covers the lens. Whether an eyelid or cornea, the tissue producing this structure in cephalopods is different from its source in vertebrates.

In addition to the differences in embryonic origin, photoreceptor cells divide into ciliary or microvillar structures to provide the membrane surface for the opsin molecule (Salvini-Plawen \& Mayr, 1977). Microvillipredominate in invertebrates while vertebrate photoreceptors are ciliary. Physiological responses are also quite different with the microvillous receptors of arthropods and mollusks depolarizing to light and the ciliary receptors of vertebrates hyperpolarizing to light. In phototransduction, vertebrate photoreceptors exploit cyclic GMP as a second messenger system while invertebrates use inositol trisphosphate (Fernald, 2000). And, even though opsin is the key molecule for detecting light, the two major classes of opsins are divided between the two groups. The response is ended quite differently between these two photoreceptor types and mechanisms for regeneration (reisomerization) of the chromophore/opsin system are dramatically different among phyla (Gonzalez-Fernandez, 2003).

\section{How did eyes evolve: independently recruited genes?}

It seems increasingly evident that as eyes evolved, different functional mechanisms have been generated by recruiting existing gene programs. Genome sequencing shows that there are far fewer genes in organisms than previously thought so increasing reports of the use and reuse of genes and their products in combinatorial assemblies make sense. In the development of eyes, this seems to be the rule not the exception. Specifically, in the evolution of eyes (see Fig. 1), it seems likely that light sensitivity evolved first, early in the Cambrian, and that this evolutionary step was in the form of a proto-opsin molecule that would ultimately be the basis of a photoreceptor cell. Since the seven trans-membrane domain protein of opsin is related to molecules used in chemoreception, photoreception possibly followed chemoreception. Importantly, the proto-opsin joined with retinal to form the basis for the large group of retinal-binding opsin photopigments known from bacteria, unicellular algae and animals. This molecular combination, sensitive to light appears to have joined forces with the genes pax6 (Sheng et al., 1997) and possibly eya (based on its phosphatase activity (Li et al., 2003)) long before there were eyes. One can imagine that this combination of a photoreceptive molecule (proto-opsin) and molecular organizers (pax6, eya) was recruited for early eyespots and other light sensing organs. It would be unsurprising, for example, to find them in the recently described eye without a nervous system (Nordström et al., 2003). Then as different eye types evolved, there was probably repeated recruitment of particular gene groups, not unlike improvisational groups of actors, interacting to produce candidates for selection. Trying out various routines could have led to numerous parallel evolutionary paths for eyes as we now envisage (Fig. 1). So the answer to the question of whether eyes evolved from a single prototypical eye (monophyletic) or if they evolved repeatedly 
(polyphyletic) may be the wrong question since it depends on the level of comparison (Land and Nilsson, 2002).

\section{Conclusions}

It is tempting to imagine that clarity about puzzling scientific issues is just around the corner even though each new level of understanding usually offers a new view of deeper complexities. Or, as Wittgenstein (1953) said: "We talk of process and states, and leave their nature undecided. Sometime perhaps we will know more about them -we think. But that is just what commits us to a particular way of looking at the matter." With relation to eye evolution, the particularities of molecular discoveries has focused attention on commonalities across eye types, often overlooking the large differences in eye structures and developmental origins. That opsin is homologous across many different types of eyes does not make those eyes homologous. Rather, at the level of light detection, eyes have converged on a common mechanism. Other functional aspects of the eye such as the lens, etc. can come from radically different sources.

Where and how progress might be expected given our current knowledge? It seems likely that as more genes and their roles are identified we will have a more refined view of eye development and evolution. A better understanding of genes expressed in the development of compound ciliary eyes and microvillar chambered eyes (see Fig. 1) would help clarify the gene recruitment processes that important for their evolution. Understanding what makes eyes so remarkably different may ultimately be a bigger challenge than discovering what they have in common.

\section{References}

ARIKAWA K, SUYAMA D, FUJII D (1996) Light on butterfly mating. Nature 382:119. BAUMER N, MARQUARDT T, STOYKOVA A, SPEILER D, TREICHEL D, ASHERYPADAN R, GRUSS P (2003) Retinal pigmented epithelium determination requires the redundant activities of Pax2 and Pax6. Development 130:2903-2925.

CHAUHANBK, REED NA, YANG Y, CERMAKL, RENEKERL, DUNCANMK, CVEKL A (2002) A comparative cDNA microarray analysis reveals a spectrum of genes regulated by Pax6 in mouse lens. Genes to Cel/s 7:1267-1283.

CHOW RL, ALTMANN CR, LANG RA, HEMMATI-BRIVANLOU A (1999) Pax-6 induces ectopic eyes in a vertebrate. Development 126:4213-4222.

CHOW RL, LANG RA (2001) Early eye development in vertebrates. Annu Rev Cell Dev Bio/17:255-296.

CONWAY-MORRIS S (1998) The Crucible of Creation. Oxford: Oxford University Press.

DOOLITTLE RF (1988) More molecular opportunism. Nature 336:18

FERNALD RD (1988) Aquatic adaptations in fish eyes. In Sensory Biology of Aquatic Animals (Atema J, Fay RR, Popper AN, Tavolga WN, eds), pp 185-208. New York: Springer Verlag.

FERNALD RD (2000) Evolution of eyes. Curr Opinion in Neurobiology 10:444-450.

FULLER RC, FLEISHMAN LJ, LEAL M, TRAVIS J, LOEW E (2003) Intra specific variation in retinal cone distribution in the bluefin killifish, Lucania goodei. J Comp Physio/A 189:609-616.

GONZALEZ-FERNANDEZ R (2003) Interphotoreceptor retinoid-binding protein: an old gene for new eyes. Vision Res 43:3021-3036.

GRAW J (1996) Genetic aspects of embryonic eye development in vertebrates. Dev Gen 18:181-197.

GRAW J (2003) The genetic and molecular basis of congenital eye defects. Nature Reviews Genetics 4:876-888.

HALDER G, CALLAERTS P, GEHRING WJ (1995) Induction of ectopic eyes by targeted expression of the eyeless gene in Drosophila. Science 267:1788-1792.
HARLAND R (2000) Neural Induction. Curr Opin in Genet Dev 10:357-362.

JACOBS GH (1996) Primate photopigments and primate color vision. Proc Nat/ Acad SCi USA 93:577-581.

KUMAR JP (2001) Signalling pathways in Drosophila and vertebrate retinal development. Nature Reviews Genetics 2:846-857.

LAND MF (1981) Opics and vision in invertebates. In Handbook of sensory physiology (Autrum H, ed), pp 471-592. Berlin: Springer.

LAND MF, FERNALD RD (1992) The Evolution of Eyes. Ann Rev Neurosci15:1-29. LAND MF, NILSSON D-E (2002) Animal Eyes. Oxford: Oxford University Press.

LI X, OGHI KA, ZHANG J, KRONES A, BUSH KT, GLASS CK, NIGAM SK, AGGARWAL AK, MAAS R, ROSE DW, ROSENFELD MG (2003) Eya protein phosphatase activity regulate Six1-Dach-Eya transcriptional effects in mammalian organogenesis. Nature 426:247-253.

LUCAS PW, DOMINY NJ, RIBA-HERNANDEZ P, STONER KE, YAMASHITA N, LORIA-CALDERON E, PETERSEN-PEREIRA W, ROJAS-DURAN Y, SALASPENA R, SOLIS-MADRIGAL S, OSORIO D, DARVELL BW (2003) Evolution and function of routine trichromatic visionin primates. Int $\mathrm{J}$ Org Evolution 57:2636-2643.

NAGEL MG, OSORIO D (1993) The tuning of human photopigments may minimize red-green chromatic signals in natural conditions. Proc R Soc LondB 252:209213.

NEITZ J, NEITZ M, KAINZ PM (1996) Visual pigment gene structure and the severity of color vision defects. Science 274:801-804.

NIEUWKOOP PD (1963) Pattern formation in artifically activated ectoderm (Rana pipens \& Ambystoma punctatum). Dev Bio/7:255-279.

NILSSON DE (1996) Eye ancestry: old genes for new eyes. Curr Bio/6:39-42.

NILSSON DE, PELGER S (1994) A pessimistic estimate of the time required for an eye to evolve. Proc $R$ Soc Lond B Biol Sci256:53-58.

NORDSTRÖM K, WALLEN R, SEYMOUR J, NILSSON D-E (2003) A simple visual system without neurons in jellyfish larvae. Proc $R$ Soc Lond B 270:2349-2354.

OAKLEY TH, CUNNINGHAM CW (2002) Molecular phylogenetic evidence for the independent evolutionary origin of an arthropod compound eye. Proc Nat/Acad SCi USA 99:1426-1430.

OKANO T, KOIMA D, FUKADA Y, SHICHIDA Y, YOSHIZAWA T (1992) Primary structures of chicken cone visual pigments: Vertebrate rhodopsins have evolved out of cone visual pigments. Proc Nat/ Acad Sci USA 89:5932-5936.

OSORIO D, VOROBYEV M (1996) Colour vision as an adaptation to frugivory in primates. Proc $R$ Soc LondB 263:593-599.

PACKARD A (1972) Cephalopods and fish: the limits of convergence. Bio Rev 47:241-307.

PARKER AR (1998) Colour in Burgess Shale animals and the effect of light on evolution in the Cambrian. Proc Royal Soc London B Biol. Sci. 265:967-972.

PIATIGORSKY, J (2003) Crystallin genes: specialization by changes in gene regulation may precede gene duplication. J. Struct. Funct. Genomics 3:131-7.

PICHAUD F, TREISMAN J, DESPLAN C (2001) Reinventing a common strategy for patterning the eye. Cel/ 105:9-12.

SALVINI-PLAWEN LV, MAYR E (1977) On the evolution of photoreceptors and eyes. Evol Biol 10:207-263.

SHENG G, THOUVENOT E, SCHMUCKER D, WILSON DS, DESPLAN C (1997) Direct regulation of rhodopsin 1 by Pax-6/eyeless in Drosophila: evidence fo ra conserved function in photoreceptors. Genes Dev 11:1122-1131.

SPEMANN H (1924) Über Organisatoren in der tierischen Entwicklung. Naturwissenschaften 48: 1092-1094.

VILTALA J, KORPIMAKI E, PALOKANGAS P, KOIVULA M (1995) Attraction of kestrels to vole scent marks visible in ultraviolet detection. Nature 373:425-427.

VOROBYEV M (2003) Coloured oil droplets enhance colour discrimination. Proc $R$ Soc Lond B270:1255-1261.

WALLS GL (1942) The vertebrate eye and its adaptive radiation. Bloomingon Hills: Cranbrook Institute.

WISTOW G (1993) Lens crystallins gene recruitment and evolutionary dynamism. Trends in Biochem Sci18:301-306.

WITTGENSTEIN L (1953) Philosophical investigations. Oxford: Basil Blackwell. 